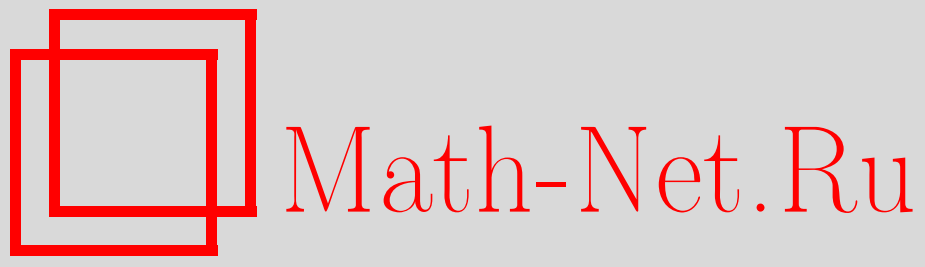

С. Н. Лакаев, Ж. И. Абдуллаев, Спектральные свойства разностного трехчастичного оператора Шрёдингера, Функи. анализ и его прил., 1999, том 33, выпуск 2, 84-88

DOI: https://doi.org/10.4213/faa359

Использование Общероссийского математического портала MathNet.Ru подразумевает, что вы прочитали и согласны с пользовательским соглашением

http://www.mathnet.ru/rus/agreement

Параметры загрузки:

IP: 54.237 .206 .68

26 апреля 2023 г., $16: 26: 30$

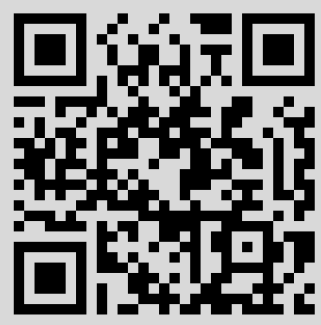




\title{
ЛитеРАТУРА
}

1. Березанский Ю. М., Ус Г. Ф., ШІефтель З. Г. Функциональный анализ. Выща школа, Киев, 1990. 2. Albeverio S., Koshmanenko V. Form-sum approximations of singular perturbations of self-adjoint operators. BiBoS Preprint Nr. 771/4/97, 1997. 3. Кошманенко В. Д. Сингулярные билинейные формы в теории возмущений самосопряженных операторов. Наукова думка, Киев, 1993. 4. Albeverio S., Karwowski W., Koshmanenko V. Math. Nachr., 173, 5-24 (1995). 5. Березанский Ю. М. В кн.: Спектральный анализ дифференциальных операторов. Институт математики, Киев, 1980, с. 83-106. 6. Крейн М. Г., Яврян В. А. J. Operator Theory, 6, 155-191 (1981). 7. Albeverio S., Koshmanenko $V$. On the problem of the right Hamiltonian under singular form-sum perturbations. SFB 237 Preprint, Nr. 375, Institute für Mathematik Ruhr-Universität-Bochum, 1997. 8. Gesztesy F., Simon B. J. Funct. Anal., 128, 245-252 (1995). 9. Albeverio S., Koshmanenko $V$. Some remarks on the Gesztesy-Simon version of rank-one perturbations. BiBoS Preprint, Nr. 727/5/96, 1996. 10. Ахиезер Н. И., Глазман И. М. Теория линейных операторов в гильбертовом пространстве. М., 1966. 11. Крейн М. Г. Матем. сб., 20, № 3, 431-495 (1947). 12. Бирман М. Ш. Матем. сб., 38 (80), № 4, 431-450 (1956). 13. Alonso A., Simon B. J. Operator Theory, 4, 251-270 (1980). 14. Яфаев Д. Р. Математическая теория рассеяния. Санкт-Петербургский университет, С.-Петербург, 1994.

Институт математики НАН Украины, Киев e-mail: kosh@imath.kiev.ua

Поступило в редакцию 15 января 1998 г.

\section{Спектральные свойства разностного трехчастичного оператора Шрёдингера*}

\author{
(c) 1999. С. Н. ЛАКАев, Ж. И. АБдУЛЛАев
}

1. Введение. В спектральном анализе трехчастичного непрерывного оператора Шрёдингера имеется следующий замечательный результат: если в системе трех частиц, взаимодействующих с помощью парных короткодействующих потенциалов, ни одна из трех двухчастичных подсистем не имеет связанных состояний с отрицательной энергией, но по меньшей мере две из них имеют резонанс с энергией в нуле, то у этой трехчастичной системы существует бесконечное число трехчастичных связанных состояний с отрицательной энергией, накапливающихся к нулю. Этот эффект впервые был обнаружен Ефимовым [1]. Строгое математическое доказательство существования эффекта Ефимова было проведено впервые в работе Яфаева [2].

В моделях физики твердого тела [3], а также решетчатой теории поля [4] возникают так называемые дискретные операторы Шрёдингера, являющиеся решетчатым аналогом обычного трехчастичного оператора Шрёдингера в непрерывном пространстве.

Хотя дискретный оператор Шрёдингера ограничен и возмущение в парной задаче - компактный оператор, спектр дискретного оператора для системы трех квантовых решетчатых частиц имеет более сложный характер по сравнению с непрерывным случаем. 
В непрерывном случае из полного гамильтониана можно выделить энергию движения центра масс так, что трехчастичные «связанные состояния» суть собственные векторы оператора энергии с отделенным полным импульсом (при этом такой оператор фактически не зависит от значений полного импульса).

Поэтому эффект Ефимова имеет или же не имеет места одновременно для всех значений полного импульса. На решетке «выделению центра масс» системы отвечает реализация гамильтониана как «расслоенного оператора», т.е. прямого интеграла семейства операторов $H_{\mu}(K)$ (где $\mu$ - энергия взаимодействия двух частиц), зависящих от значений полного квазиимпульса $K \in T^{3}$, $T^{3}$ — трехмерный тор. В этом случае «связанное состояние» есть собственный вектор оператора $H_{\mu}(K)$ для некоторого $K \in T^{3}$, в типичном случае непрерывно меняющийся при изменении $K$. В работе [5] рассмотрен дискретный оператор Шрёдингера, описывающий системы трех одинаковых квантовых частиц (бозонов), взаимодействующих с помощью парных контактных потенциалов притяжения при общих предположениях на энергии отдельного бозона $\varepsilon(q), q \in T^{3}$. В этой работе доказано только существование бесконечного числа собственных значений, лежащих левее существенного спектра, при значениях полного квазиимпульса $K$, которые принадлежат $M$, где $M$ многообразие коразмерности 1 в торе $T^{3}$.

В настоящей работе мы рассмотрим разностный оператор Шрёдингера, т. е. $\varepsilon(q)=\sum_{i=1}^{3}\left(1-\cos q_{i}\right)$, и покажем, что только при нулевом значении полного квазиимпульса $(K=0)$ и некотором $\mu=\mu_{0}>0$ имеет место эффект Ефимова, а при всех остальных значениях $K \in T^{3}$ и $\mu>0$ оператор $H_{\mu}(K)$ имеет лишь конечное число собственных значений, лежащих вне существенного спектра.

Это явление, т. е. такая нетривиальная зависимость от квазиимпульса системы, позволяет следующим образом сформулировать эффект Ефимова для систем квантовых частиц на решетке: если левые края «трехчастичной» и «двухчастичной» ветвей существенного спектра совпадают и этот край является виртуальным уровнем оператора $h_{\mu}(2 K / 3)+\varepsilon(K / 3) I$, где $h_{\mu}(k)$ - двухчастичный оператор, а $I$ - единичный оператор, то у трехчастичного оператора $H_{\mu}(K)$ существует бесконечное число собственных значений, лежащих левее существенного спектра.

Авторы выражают глубокую благодарность Р. А. Минлосу за внимание и обсуждения полученных результатов, а также рецензенту за ценные замечания.

2. Постановка задачи и формулировка результатов. Пусть $\mathbb{Z}^{3}$ трехмерная решетка, $\ell_{2}\left(\left(\mathbb{Z}^{3}\right)^{m}\right)$ - гильбертово пространство квадратично суммируемых функций, определенных на $\left(\mathbb{Z}^{3}\right)^{m}$, и $\ell_{2}^{s}\left(\left(\mathbb{Z}^{3}\right)^{m}\right) \subset \ell_{2}\left(\left(\mathbb{Z}^{3}\right)^{m}\right)$ подпространство, состояшее из функций $\psi\left(n_{1}, \ldots, n_{m}\right)$, симметричных относительно перестановки любых двух переменных.

В координатном представлении дискретные операторы Шрёдингера системы двух одинаковых частиц и системы трех одинаковых частиц на трехмерной решетке действуют соответственно в пространствах $\ell_{2}^{s}\left(\left(\mathbb{Z}^{3}\right)^{2}\right)$ и $\ell_{2}^{s}\left(\left(\mathbb{Z}^{3}\right)^{3}\right)$ по формулам 


$$
\begin{aligned}
\left(\tilde{h}_{\mu} \varphi\right)\left(n_{1}, n_{2}\right)= & \frac{1}{2} \sum_{|s|=1}\left[2 \varphi\left(n_{1}, n_{2}\right)-\varphi\left(n_{1}+s, n_{2}\right)-\varphi\left(n_{1}, n_{2}+s\right)\right] \\
& -\mu \delta_{n_{1} n_{2}} \varphi\left(n_{1}, n_{2}\right), \\
\left(\widetilde{H}_{\mu} \varphi\right)\left(n_{1}, n_{2}, n_{3}\right)= & \frac{1}{2} \sum_{|s|=1}\left[3 \varphi\left(n_{1}, n_{2}, n_{3}\right)-\varphi\left(n_{1}+s, n_{2}, n_{3}\right)-\varphi\left(n_{1}, n_{2}+s, n_{3}\right)\right. \\
& \left.-\varphi\left(n_{1}, n_{2}, n_{3}+s\right)\right]-\mu\left(\delta_{n_{1} n_{2}}+\delta_{n_{1} n_{3}}+\delta_{n_{3} n_{2}}\right) \varphi\left(n_{1}, n_{2}, n_{3}\right) .
\end{aligned}
$$

Здесь $\mu>0$ - энергия взаимодействия, а $\delta_{n m}$ - символ Кронекера.

Пусть $T^{3}$ - трехмерный тор и $L_{2}^{s}\left(\left(T^{3}\right)^{m}\right)$ - гильбертово пространство квадратично интегрируемых симметричных функций, определенных на $\left(T^{3}\right)^{m}$. В импульсном представлении дискретные операторы Шрёдингера системы двух одинаковых частиц и системы трех одинаковых частиц действуют соответственно в гильбертовых пространствах $L_{2}^{s}\left(\left(T^{3}\right)^{2}\right)$ и $L_{2}^{s}\left(\left(T^{3}\right)^{3}\right)$ по формулам

$$
\begin{aligned}
\left(h_{\mu} f\right)\left(k_{1}, k_{2}\right)=\left(\varepsilon\left(k_{1}\right)+\right. & \left.\varepsilon\left(k_{2}\right)\right) f\left(k_{1}, k_{2}\right) \\
& -\mu \int_{\left(T^{3}\right)^{2}} \delta\left(k_{1}+k_{2}-k_{1}^{\prime}-k_{2}^{\prime}\right) f\left(k_{1}^{\prime}, k_{2}^{\prime}\right) d k_{1}^{\prime} d k_{2}^{\prime},
\end{aligned}
$$

$\left(H_{\mu} f\right)\left(k_{1}, k_{2}, k_{3}\right)=\left(\varepsilon\left(k_{1}\right)+\varepsilon\left(k_{2}\right)+\varepsilon\left(k_{3}\right)\right) f\left(k_{1}, k_{2}, k_{3}\right)$

$$
-\mu \sum_{\alpha=1}^{3} \int_{\left(T^{3}\right)^{3}} \delta\left(k_{\alpha}-k_{\alpha}^{\prime}\right) \delta\left(k_{\beta}+k_{\gamma}-k_{\beta}^{\prime} k_{\gamma}^{\prime}\right) f\left(k_{1}^{\prime}, k_{2}^{\prime}, k_{3}^{\prime}\right) d k_{1}^{\prime} d k_{2}^{\prime} d k_{3}^{\prime} .
$$

Здесь $\alpha<\beta<\gamma, \alpha, \beta, \gamma=1,2,3$,

$$
\varepsilon(p)=\sum_{i=1}^{3}\left(1-\cos p_{i}\right), \quad p=\left(p_{1}, p_{2}, p_{3}\right) \in T^{3},
$$

и $\delta(k)$ - трехмерная дельта-функция Дирака. На торе $T^{3}$ выбрана единичная мера, т.е. $\int_{T^{3}} d k=1$.

Пусть $k=k_{1}+k_{2}$ и $K=k_{1}+k_{2}+k_{3}$ - полные квазиимпульсы системы двух частиц и системы трех частиц, а $\Gamma_{k}=\left\{\left(k_{1}, k_{2}\right) \in\left(T^{3}\right)^{2}: k=k_{1}+k_{2}\right\}$ и $F_{K}=\left\{\left(k_{1}, k_{2}, k_{3}\right) \in\left(T^{3}\right)^{3}: K=k_{1}+k_{2}+k_{3}\right\}$ - соответственно трехмерное и шестимерное многообразия. Обозначим через $\tilde{L}(k)$ и $L(K)$ гильбертовы пространства всех квадратично интегрируемых функций, определенных на $T^{3}$ и $\left(T^{3}\right)^{2}$ и удовлетворяющих соответственно условиям $f\left(k_{1}\right)=f\left(k-k_{1}\right)$ и $f\left(k_{1}, k_{2}\right)=f\left(k_{2}, k_{1}\right)=f\left(k_{1}, K-k_{1}-k_{2}\right)$.

Операторы $h_{\mu}$ и $H_{\mu}$ разлагаются в прямые интегралы [5]

$$
h_{\mu}=\int_{T^{3}} \oplus h_{\mu}(k) d k, \quad H_{\mu}=\int_{T^{3}} \oplus H_{\mu}(K) d K,
$$

где ограниченные самосопряженные операторы $h_{\mu}(k), H_{\mu}(K), k, K \in T^{3}$ действуют в гильбертовых пространствах $\tilde{L}(k)$ и $L(K)$ соответственно по формулам

$$
\begin{aligned}
\left(h_{\mu}(k) f\right)(p) & =\varepsilon_{k}(p) f(p)-\mu \int_{T^{3}} f(s) d s, \\
\left(H_{\mu}(K) f\right)(p, q) & =\varepsilon_{K}(p, q) f(p, q)-\mu \int_{T^{3}}[f(K-p-q, s)+f(p, s)+f(s, q)] d s .
\end{aligned}
$$

Здесь $\varepsilon_{k}(p)=\varepsilon(k-p)+\varepsilon(p), \varepsilon_{K}(p, q)=\varepsilon(K-p-q)+\varepsilon(p)+\varepsilon(q)$. 
Для любого $k \in T^{3}$ положим

$$
\mu^{-1}(k)=\int_{T^{3}} \frac{d q}{\varepsilon_{k}(q)-\varepsilon(k / 2)} .
$$

Из монотонности интеграла Лебега следует, что $\max _{k \in T^{3}} \mu(k)=\mu(0)=\mu_{0}$.

ЛЕммА. Для любого $\mu>0$ существует открытое связное множество $G_{\mu}$, такое, что для любого $k \in G_{\mu}$ оператор $h_{\mu}(k)$ имеет единственное простое собственное значение $z=z_{\mu}(k)$, лежащее левее его непрерьвного спектра, и ни при каких $k \in T^{3} \backslash G_{\mu}$ оператор $h_{\mu}(k)$ не имеет собственных значений вне непрерывного спектра. При этом если $\mu>\mu_{0}$, то $G_{\mu}=T^{3}$.

Пусть $E_{\mu, K}(q)=z_{\mu}(K-q)+\varepsilon(q)$. Положим

$$
\begin{aligned}
\varepsilon_{\min }(K) & =\min _{p, q \in T^{3}} \varepsilon_{K}(p, q), & \varepsilon_{\max }(K) & =\max _{p, q \in T^{3}} \varepsilon_{K}(p, q), \\
E_{\min }(\mu, K) & =\min _{q} E_{\mu, K}(q), & E_{\max }(\mu, K) & =\max _{q} E_{\mu, K}(q) .
\end{aligned}
$$

Теорема 1. Существенный спектр $\sigma_{\mathrm{ess}}\left(H_{\mu}(K)\right)$ оператора $H_{\mu}(K)$ совпадает с множеством

$$
\left[E_{\min }(\mu, K), E_{\max }(\mu, K)\right] \cup\left[\varepsilon_{\min }(K), \varepsilon_{\max }(K)\right] .
$$

ТЕОрема 2. Ни для какого $\mu>0$ оператор $H_{\mu}(K)$ не имеет собственных значений, лежащих правее его существенного спектра.

Tеорема 3. (а) Пусть $K=0, \mu=\mu_{0}$. Тогда оператор $H_{\mu_{0}}(0)$ имеет бесконечное число отрицательных собственных значений $E_{1}, \ldots, E_{n}, \ldots$ При этом $\lim _{n \rightarrow \infty} E_{n}=0$.

(б) Пусть $K \neq 0$; тогда для любого $\mu>0$ дискретный спектр оператора $H_{\mu}(K)$ не более чем конечен.

СХема доказАтельствА. Пусть $T_{\mu, K}(z)$ - оператор Фаддеева, отвечающий уравнениям фаддеевского типа на решетке (см. [5]), и пусть $\widehat{H}_{\mu}(K)-$ оператор канала, соответствующий двум взаимодействующим и одной свободной частицам. При $z \in C \backslash \sigma\left(\widehat{H}_{\mu}(K)\right)$ определен определитель Фредгольма оператора $I-T_{\mu, K}(z)$, причем в области $C \backslash \sigma\left(\widehat{H}_{\mu}(K)\right)$ он является регулярной функцией от $z$. Поэтому этот определитель в $C \backslash \sigma\left(\widehat{H}_{\mu}(K)\right)$ имеет не более чем счетное число нулей. Отсюда и из определения существенного спектра следует утверждение теоремы 1.

Из положительности возмущения $H_{0}(K)-H_{\mu}(K)$ и принципа минимакса следует утверждение теоремы 2.

Утверждение (а) теоремы 3 основывается на исследовании непрерывного спектра предельного оператора $T_{\mu_{0}, 0}(0)$. Утверждение (б) следует из компактности предельного оператора $T_{\mu, K}(Z(K))$, где

$$
Z(K)=\min \left\{E_{\min }(\mu, K), \varepsilon_{\min }(K)\right\},
$$

в рассматриваемом гильбертовом пространстве или же в подходящим образом выбранном банаховом пространстве.

\section{ЛИТЕРАТУРА}

1. Ефимов В. Н. Ядерная физика, 12, вып. 5, 1080-1091 (1970). 2. Яфаев Д. Р. Матем. сб., 9(136), № 4(8). 567-592 (1974). 3. Mattis D. C. Rev. Modern Phys., 58, No. 2, 
361-379 (1986). 4. Мальшев В. А., Минлос Р. А. Труды семинара им. И. Г. Петровского, вып. 9, 63-80 (1983). 5. Лакаев C. Н. Функц. анализ и его прил., 27, вып. 3, $15-28$ (1993).

Самаркандский государственный университет

Поступило в редакцию 10 ноября 1997 г.

\section{УДК 517.98}

\section{Псевдодифференциальные операторы на многообразиях с особенностями}

\section{(c) 1999. Б. А. ПлАменевСкий, В. Н. СЕничкин}

В заметке определяются ПДО на многообразиях с «пересекающимися ребрами»; перечисляются неприводимые представления $C^{*}$-алгебры, порожденной ПДО (нулевого порядка).

1. Стратифицированные многообразия. Компактное метрическое пространство $\mathscr{M}$ назовем стратифичированнылм многообразием, если: 1) $\mathscr{M}$ есть конечное объединение непересекающихся связных топологических многообразий (cmpamoв); 2) граница $\partial T=\bar{T} \backslash T$ каждого страта $T$ есть объединение некоторого (возможно, пустого) множества стратов меньших размерностей. По определению $\operatorname{dim} \mathscr{M}$ есть наибольшая из размерностей стратов. Множество всех стратов, границы которых содержат страт $T$, называется звездой st $T$ страта $T$. Последовательность $T_{0}, T_{1}, \ldots, T_{p}$ стратов называется чепочкой, если $T_{k} \in$ st $T_{j}$ при $k>j$. Определим дифференциальную структуру класса $\mathfrak{D}_{n}$. Через $\mathfrak{D}_{0}$ обозначим множество конечных наборов точек с дискретной топологией. Пусть определены $\mathfrak{D}_{k}, 0 \leqslant k \leqslant n-1$. Многообразие $\mathscr{M}$ класса $\mathfrak{D}_{n}(n=\operatorname{dim} \mathscr{M})$ подчиняется следующему условию: для любого страта $T, \operatorname{dim} T=q$, и всякой точки $x \in T$ существуют окрестность $\mathscr{O}$ точки $x$ в $\mathscr{M}$ и гомеоморфизм $\kappa: \mathscr{O} \rightarrow \kappa(\mathscr{O})$, такой, что $\kappa(\mathscr{O})$ - открытое подмножество произведения $\mathbb{R}^{q} \times K\left(\mathscr{M}^{\prime}\right)$, где $K\left(\mathscr{M}^{\prime}\right)$ замкнутый конус в некотором пространстве $\mathbb{R}^{N}$ с направляющим многообразием $\mathscr{M}^{\prime} \in \mathfrak{D}_{n-q-1}$. При этом $\kappa(T \cap \mathscr{O}) \subset \mathbb{R}^{q} \times 0,0$ - вершина конуса $K\left(\mathscr{M}^{\prime}\right)$, и если $R \in \operatorname{st} T, \operatorname{dim} R=r$, то $\kappa(R \cap \mathscr{O}) \subset \mathbb{R}^{q} \times K\left(R^{\prime}\right)$, где $R^{\prime}$ есть $(q-r-1)$-мерный страт многообразия $\mathscr{M}^{\prime}$. (Будем говорить, что $R^{\prime}$ отвечает страту $R$.) Предположим, что $c_{1} r\left(a_{1}, a_{2}\right) \leqslant \operatorname{dist}\left(a_{1}, a_{2}\right) \leqslant c_{2} r\left(a_{1}, a_{2}\right)$ для всех $a_{1}, a_{2} \in \mathscr{O}$, где $r$ - расстояние на $\mathscr{M}$, dist - расстояние в $\mathbb{R}^{q+N}$ и $c_{1}, c_{2}$ - положительные постоянные. В окрестности $\mathscr{O}$ введем «цилиндрические» координаты $\left(\zeta_{1}, \rho_{1}, \omega_{1}\right)$ при $\zeta_{1} \in \mathbb{R}^{q}, \rho_{1}>0, \omega_{1} \in \mathscr{M}^{\prime}$. Полагая $q_{1}=q$ и «расшифровывая» $\omega_{1}$ по индукции, получаем координатную окрестность $U$ с цилиндрическими координатами $\left(\zeta_{1}, \rho_{1}, \ldots, \zeta_{k}, \rho_{k}, \zeta_{k+1}\right)$, где $\zeta_{j} \in \mathbb{R}^{q_{j}}$, $\rho_{j}>0$; не исключается случай $q_{j}=0$ (тогда $\zeta_{j}$ отсутствует). Введем вектор $\left(\zeta_{1}, \rho_{1}, \rho_{1} \zeta_{2}, \rho_{1} \rho_{2}, \rho_{1} \rho_{2} \zeta_{3}, \rho_{1} \rho_{2} \rho_{3}, \ldots, \rho_{1} \ldots \rho_{k-1} \zeta_{k}, \rho_{1} \ldots \rho_{k-1} \rho_{k}, \rho_{1} \ldots \rho_{k} \zeta_{k+1}\right)$ и перепишем его как числовую строку $\left(x_{1}, \ldots, x_{n}\right)$; она играет роль декартовых координат. Будем считать, что цилиндрические координаты в пересекающихся окрестностях связаны гладким преобразованием (в том числе и при нулевых значениях координат $\rho_{j}$ ). Предположим, что из окрестностей типа окрестности $U$ составлен конечный атлас $\left\{U_{i}, \kappa_{i}\right\}$. Атлас с перечисленными 